\title{
K-ras in Colorectal Cancer Tumors From Saudi Patients: Frequency, Clinco-pathological Association and Clinical Outcome
}

\author{
Jamal Zekri*, ${ }^{, 1}$, Azhar Rizvi ${ }^{1}$, Jaudah Al-Maghrabi ${ }^{2,3}$, and Baker bin Sadiq ${ }^{4}$ \\ ${ }^{I}$ Department of Oncology, King Faisal Specialist Hospital \& Research Centre, Jeddah, Saudi Arabia \\ ${ }^{2}$ Department of Pathology, King Abdulaziz University, Jeddah, Saudi Arabia \\ ${ }^{3}$ Department of Pathology, King Faisal Specialist Hospital \& Research Centre, Jeddah, Saudi Arabia \\ ${ }^{4}$ Research Department, King Faisal Specialist Hospital \& Research Centre, Jeddah, Saudi Arabia
}

\begin{abstract}
Background: K-ras oncogene mutations are confirmed factor for lack of clinical benefit from antibodies targeting EGFR in patients with colorectal cancer (CRC). Mutations are reported in 40\% of CRC tumors in western patients. There is scarcity of data on population from Asia and the Middle East.

Aims: This study investigates the frequency and impact of k-ras mutation and the association between clinico-pathological features and K-ras status in Saudi patients with CRC.

Patients and Methods: Retrospective review of K-ras status, clinico-pathological characteristics and clinical outcome in 46 patients with CRC.

Results: K-ras mutations were identified in 15/46 (32\%) tumors, $87 \%$ at codon 12 and $13 \%$ at codon 13 . Gender, site of tumor, stage at diagnosis, differentiation and lymphatic and vascular invasion were tested as potential risk predictors for K-ras status. Only gender was found to be a potential risk factor. Female gender compared to male posed higher significant chance of wild type status ( $\mathrm{RR}=1.54,65 \% \mathrm{CI}: 1.07-2.2 ; \mathrm{P}=0.034)$. K-ras status (mutant vs. wild) did not statistically significantly impact on clinical outcome as measured by development of relapsed disease ( $80 \%$ vs. $81 \%)$, median relapse free survival (17 vs. 11 months, $\mathrm{P}=0.256$ ) and overall survival (not reached in both groups, $\mathrm{P}=0.59$ ).

Conclusion: This relatively small retrospective series shows that rate of K-ras mutation in Saudi patients with CRC is lower than reported in western Caucasian population but close to rates reported in neighboring Asian population. Mutations are less frequent in females. In these patients, K-ras mutation status did not significantly impact clinical outcome.
\end{abstract}

Keywords: Asia, Colorectal cancer, K-ras, Mutant, Wild type, Saudi.

\section{INTRODUCTION}

Colorectal cancer (CRC) is the third and fourth most common cancer in women and men worldwide, respectively and the fourth commonest cause of cancer death [1]. The outcome of treatment of patients with a metastatic CRC has improved in the past decade. This improvement was mainly due to the introduction of both active new chemotherapeutic agents [2] and novel targeted drugs [3].

Cetuximab is a chimeric IgG1 monoclonal antibody with high selectivity and affinity for the epidermal growth factor receptor (EGFR), which is over-expressed by $25-80 \%$ of colorectal cancer tumors [4, 5]. Clinically, cetuximab demonstrated the ability to evade chemotherapy resistance [6] and improved response rate (RR) and progression free

\footnotetext{
*Address correspondence to this author at the Department of Oncology, King Faisal Specialist Hospital \& Research Centre Jeddah, Saudi Arabia; Tel: 00966266 77777, Ext: 65066; Fax: 00966266 77777, Ext: 64030;

E-mail: jmzekri@hotmail.com
}

survival (PFS) when combined with FOLFIRI in the first line setting [7].

Until recently, the most consistent predictor for response and survival to cetuximab is the development of skin rash [6]. K-ras (Kirsten-ras) oncogene mutation occurs in 58\% of colonic adenomas larger than $1 \mathrm{~cm}$ and in $47 \%$ of CRC [8].

The K-ras gene encodes the K-ras protein that regulates 2 signaling pathways: PI3K/AKT and RAF/MEK/ERK. These pathways control cell proliferation, differentiation and apoptosis. Most frequent mutations are in codons 12 and 13 from exon 2. Over the last few years, increasing number of reports confirmed the correlation between K-ras gene status (mutant vs. wild type) and clinical benefit from cetuximab [9]. Now cetuximab based therapy is indicated only in patients with tumors expressing wild type K-ras genotype.

Collectively four randomized studies included a total of 2,292 mostly western patients with known k-ras status. 1,389 $(60 \%)$ and $903(40 \%)$ patients had wild and mutated K-ras tumors respectively [9]. Most of data on frequency and impact of k-ras status is reported on Western population and 
there scarcity of data on population from Asia and the Middle East.

The aim of this retrospective study is to investigate the frequency and impact of k-ras mutation at codons 12 and 13 and the association between clinico-pathological features and K-ras status exclusively in Saudi patients with CRC.

\section{MATERIALS AND METHODOLOGY}

46 consecutive CRC samples from 46 Saudi patients were examined for K-ras status purely for clinical indication and as requested by the treating oncologist. Patients were diagnosed with CRC with in the period of November 2003 and January 2011. The first sample in this series was tested for K-ras status in October 2009 and last in February 2011. All data were retrospectively collected by the investigators over a period of one month from 15 March 2011 until 15 April 2011.

K-ras mutation analysis was performed by Biomnis laboratory. Formalin fixed paraffin embedded material from colorectal carcinomas were used for DNA extraction. Array kit was used for detection of mutations within codons 12 and 13 of K-ras. The mutations tested are p.G12S, p.G12R, p.G-
12C, p.G12D, p.G12A, p.G12V ,p.G13D, p.G13D, p.G13R.

Tumor and patients characteristics were retrospectively extracted from paper and electronic records by 2 medical oncologists.

Clinico-pathological features selected for association testing included gender, site of tumor, AJCC stage at diagnosis, tumor differentiation, vascular invasion and lymphatic invasion. Tumors of the ascending and transverse colon were labeled as right side tumors. Tumors of the descending and sigmoid colon (without any rectal involvement) were labeled as left side tumors. Tumors extending to parts of sigmoid colon and rectum at the same time are labeled as rectosigmoid tumors. Tumors involving only the rectum are labeled as rectal tumors.

Association between clinico-pathological features and Kras status was analyzed using Chi square test. Survival tables and Kaplan Meier test were used for relapse free survival (RFS) and overall survival (OS). SPSS version 12 software was used for all analyses. Approval of institutional review board (ethics committee) has been obtained prior to conducting this study.

Table 1. Patients' Characteristics and Association between Clinico-pathological Features and K-ras Status

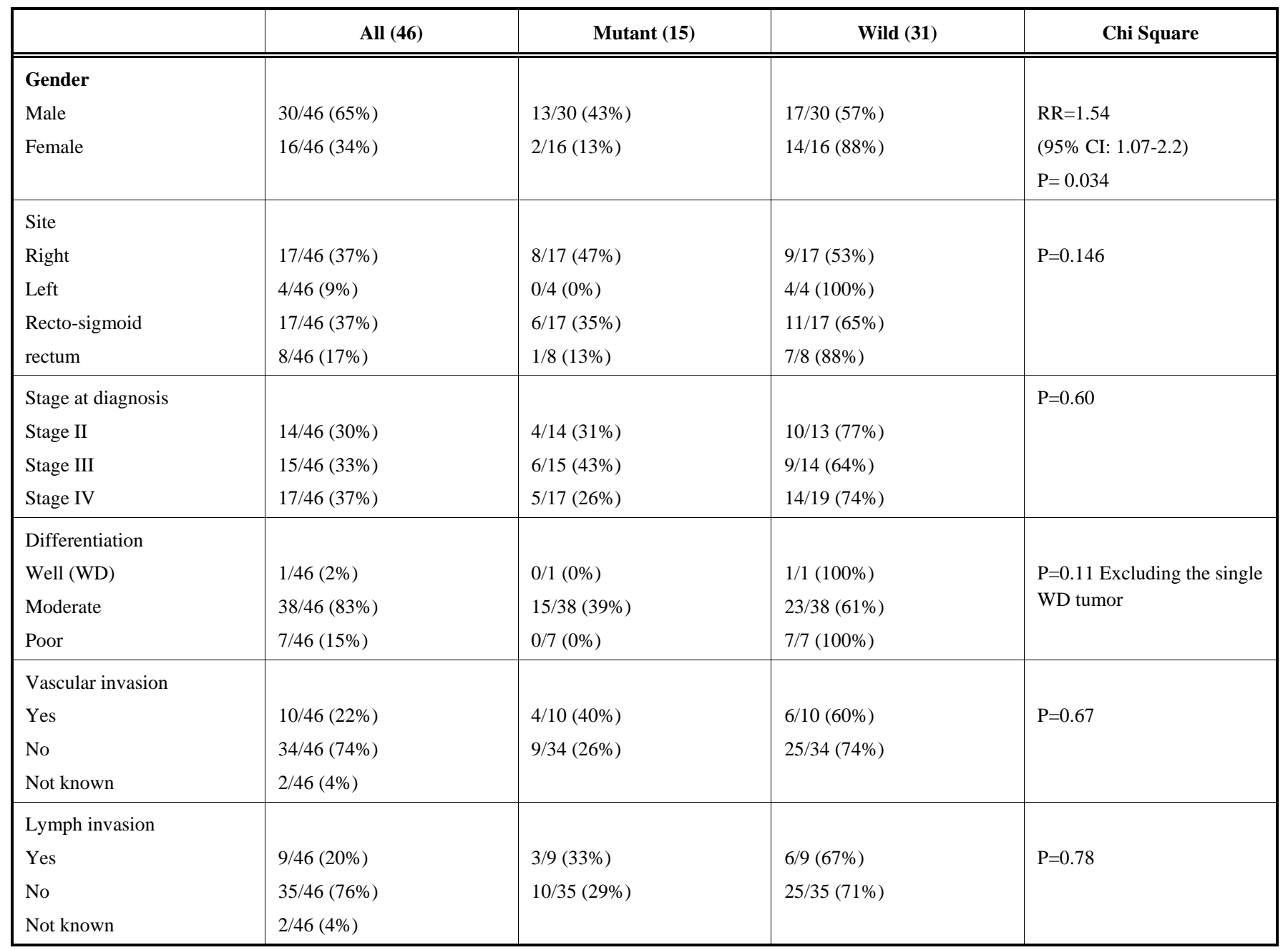




\section{RESULTS}

Thirty patients were males and 16 were females. Median age was 61 (21-80) years. Patients' characteristics are depicted in Table $\mathbf{1 .}$

K-ras mutation was identified in 15/46 (32\%) tumors. Mutations were identified in codons 12 and 13 in 13/15 (87\%), 2/15 (13\%) respectively.

Among males, $43 \%$ and $57 \%$ of tumors were mutant and wild respectively. Among females, $13 \%$ and $88 \%$ of tumors were mutant and wild respectively.

Gender, site of tumor, stage at diagnosis, tumor differentiation and lymphatic and vascular invasion were tested as potential risk predictors for K-ras status (Table 1). Gender was found to be a potential risk factor. Female gender compared to male posed higher significant chance of wild type status $(\mathrm{RR}=1.54,65 \% \mathrm{CI}: 1.07-2.2 ; \mathrm{P}=0.034)$. There was a trend for relatively higher frequency of mutant status in right side tumors $(47 \%)$ compared to other sites. However, this did not translate to statistical significance. Site of tumor, stage at diagnosis, tumor differentiation and lymphatic and vascular invasion were not statistically significant predictors for K-ras status.

Median duration of follow up for all patients was 17 (167) months.

In mutant and wild type groups, similar proportion of patients experienced relapse or metastatic disease at time of diagnosis ( $80 \%$ \& $81 \%$ respectively). For patients diagnosed with non metastatic disease (stage II/III, $n=29$ ), median RFS was 13.5 (4.7-22.2) months. RFS was not statistically different between mutant \& wild type groups (17 vs. 11 months respectively; $\mathrm{P}=0.256$ ) (Fig. 1). Median OS was not reached in both groups and was not statistically different $(\mathrm{P}=0.59)$

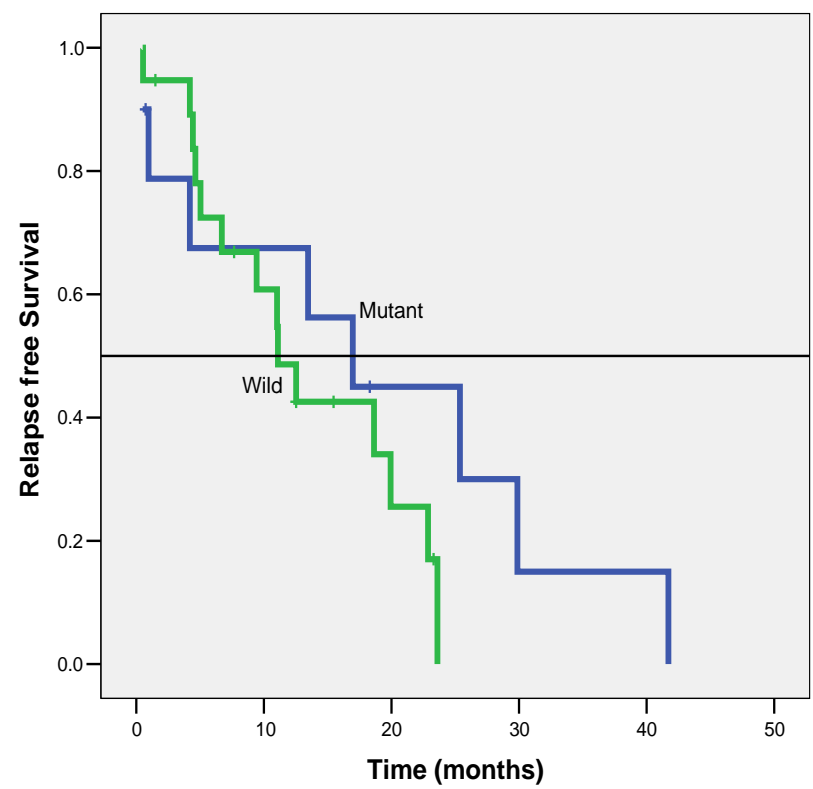

Fig. (1). Relapse Free Survival of Patients with Mutant $(n=10)$ and Wild ( $\mathrm{n}=19)$ Type K-ras. (Median RFS: $M=17 \& \mathrm{~W}=11$ months; $\mathrm{P}=0.256)$

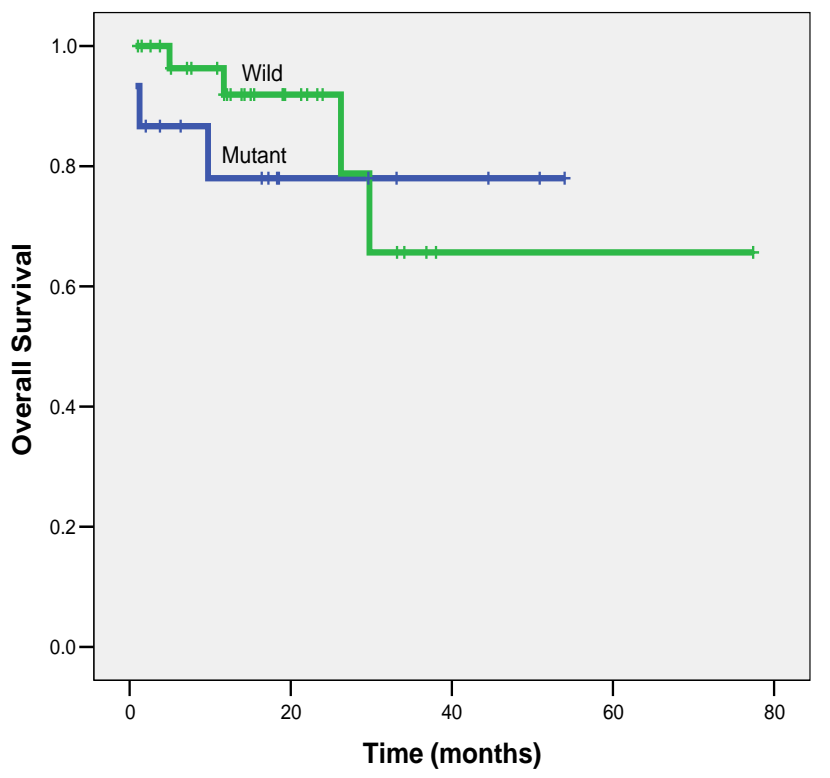

Fig. (2). Overall Survival of Patients with Mutant $(n=15)$ and Wild $(\mathrm{n}=31)$ Type K-ras. (Median OS: Not reached for both groups; $\mathrm{P}=0.59)$

(Fig. 2).

\section{DISCUSSION}

This study showed that the rate of K-ras mutation exclusively in Saudi patients with stage II/III/IV CRC is $32 \%$. This rate of K-ras mutation in this ethnic group is lower than reported in 4 large randomizes trials testing the efficacy of cetuximab in mostly western patients [10-13] (Table 2). Collectively, the average rate of mutation in these 4 studies was $40 \%$ (37\%-43\%). Similarly, in a recent large series of 2121 patients with metastatic CRC from the United States of America, the rate of K-ras mutation was 42\% [14].

Difference in rate of K-ras mutation according to geographic area of residence and ethnic origin is increasingly recognized. Clear difference in frequency of mutation was reported in one of these studies according to geographic area of recruited patients [13]. Patients recruited from Western Europe had the highest frequency of mutation followed by Eastern Europe while those from outside Europe had the lowest (Table 2).

The only other study investigating pattern of K-ras mutation in CRC Saudi patients showed a mutation rate of $28.1 \%$ [15]. Even lower rates were reported in $53 \mathrm{Kashmiri}$ patients from India (22.6\%) and 59 Egyptian patients (11\%) with CRC $[16,17]$.

These findings strongly suggest that rate of K-ras mutation in CRC is lower in Asian and Middle East population than in western and European population. This may be complicated by the extensive range of ethnic origin groups that live in Asia and the Middle East. Despite the low rates of mutation reported in Asian patients of Saudi and Kashmiri ethnic background [15,16, this study], higher rates in other Asian patients of Chinese, Israeli and Japanese ethnic 
Table.2. Frequency of K-ras Mutation in Large Randomized Studies Investigating Cetuximab in Patients with Metastatic CRC

\begin{tabular}{|l|l|l|l|}
\hline \multicolumn{1}{|c|}{ Reference and Treatment } & \multicolumn{1}{|c|}{$\begin{array}{c}\text { Number of Samples Tested/ } \\
\text { Number of Total Patients }\end{array}$} & \multicolumn{1}{|c|}{ Rate of K-ras Mutation (\%) } & $\begin{array}{c}\text { Rate of K-ras Mutation in Geo- } \\
\text { graphic Groups (\%) }\end{array}$ \\
\hline \hline [10] BSC vs. Cet & $394 / 572$ & 42.3 & NR \\
\hline [11] FOLFOX vs. FOLFOX\&Cet & $315 / 344$ & 43 & NR \\
\hline [12] XELOX\&B vs. XELOX\&B\&Cet & $528 / 755$ & 39.6 & NR \\
\hline [13] FOLFIRI vs. FOLFIRI\&Cet & $1063 / 1198$ & 37 & $\begin{array}{l}\text { Western Europe } 44.7 \\
\text { Eastern Europe 35.8 } \\
\text { Outside Europe 19.5 }\end{array}$ \\
\hline
\end{tabular}

BSC $=$ Best supportive care, $\mathrm{B}=$ Bevacizumab, Cet $=$ Cetuximab, FOLFOX $=$ Folinic acid, fluorouracil and oxaliplatin, FOLFIRI $=$ Folinic acid, fluorouracil and irinotecan, $\mathrm{XELOX}=$ Capecitabine and oxaliplatin, $\mathrm{NR}=$ Not reported.

groups; $34.7 \%, 44.8 \%$ and $42 \%$ respectively have been reported [18-20]. This suggests a probable ethnic rather than mere geographic variation.

In our study, mutations were identified in codons 12 and 13 in $87 \%$ and $13 \%$ respectively. This is similar to what is reported in the randomized phase III study comparing cetuximab and best supportive care in patients mostly from Canada and Australia were mutations were identified in codons 12 and 13 in $86.6 \%$ and $13.5 \%$ respectively [10]. This is also similar to what is reported in patients from Brazil; $87 \%$ of mutations were in codon 12 and $13 \%$ in codon 13 [21]. In studies conducted on Asian patients, mutations were identified in codons 12 and 13 in $81.2 \%$ and $18.8 \%$ in Saudi patients and in $68.3 \%$ and $29.3 \%$ in Chinese patients $[15,18]$. This indicates that codon site of mutation is not widely variable among different geographic and ethnic population.

Due to limited number of patients in our series we decided to study the association of K-ras status with only limited clinico-pathological variables. These were Gender, site of tumor, stage at diagnosis, tumor differentiation and lymphatic and vascular invasion. Our results show that Saudi females with CRC are less likely to have mutant K-ras than males. In other word; males are more likely to have mutant K-ras status than females. There are only few reports that show correlation between gender and rate of K-ras mutation. Similar finding was reported in patients from Brazil; a significant difference in the percentage of mutated K-ras was observed between male and female ( 41 vs. $35 \%, p=0.05$ ) in a cohort of 989 patients [21]. In contrast, K-ras mutation was higher in females than males $(44.7 \%$ vs. $28.2 \%, P=0.037)$ in 118 patients from China with the caveat that gender association was different according to the site of tumor [18]. In those Chinese patients, K-ras mutation frequency in the rectum was higher in female than in male patients $(34.0 \%$ vs. $11.2 \%$, $P<0.05)$ and male patients had a higher mutation rate in the colon than female patients $(16.9 \%$ vs. $10.6 \%, P<0.05)$. It should be noted that $44.1 \%$ of patients in the Chinese study had rectal cancer while only $17 \%$ in our study.

In our study and considering 4 different sites of the colorectal tract, there was a trend for relatively higher frequency of mutant status in right side tumors (47\%) compared to oth- er sites (Table 1). However, this did not translate to statistical significance.

When dividing the colorectal tract to only 2 sites; (I) right: caecum and ascending colon and (II) left: transverse, descending and sigmoid colon and rectum, there is a clear trend of higher frequency of mutation in the right colon (47\%) compared to the left colon (24\%). This is consistent with the findings in the Chinese and the larger Saudi study: mutation rate is $52 \%$ vs. $29 \%$ and $41 \%$ vs. $25.7 \%$ at right and left sites respectively $[18,15]$. A small study of 12 patients from Romania also showed a trend for higher frequency of mutation $(33.3 \%)$ in the proximal colon compared to $(16.67 \%)$ in distal colon [22].

All other clinico-pathological factors tested in our study were not found to significantly predict or associate with $\mathrm{K}$ ras status. The Chinese study showed similar finding where age, tumor location, tumor differentiation and stage did not show significant association with K-ras status [18]. Consistent with our findings, the larger Saudi study did not show signification association between K-ras status and tumor stage and differentiation [15].

European data of 404 patients from Switzerland showed partly similar findings with lack of association between Kras status and clinico-pathological parameters, such as gender, age, tumor location, histological type, tumor stage, tumor grade and vascular invasion [23].

Some studies have indicated the importance of K-ras alterations in predicting long-term outcome, while others have failed to show such a relationship.

In our study in mutant and wild type groups, similar proportion of patients experienced relapse or metastatic disease at time of diagnosis ( $80 \% \& 81 \%$ respectively) indicating that K-ras status may not predict clinical aggressiveness of disease. This is also supported by the fact that RFS for the 29 patients presented with early resectable disease (stage II/III) and OS for all patients was not statistically different between mutant and wild type groups. Our results are supported by the findings of a study of 508 cases identified among 1264 patients with stage III colon cancer who enrolled in a randomized adjuvant chemotherapy trial. Patients with mutant and wild type tumors did not experience any difference in 
RFS or OS [24]. This is in contrast to the finding in another study. The NO147 study investigated the role of adding cetuximab to adjuvant chemotherapy in 2581 patients with stage III colon cancer. Cetuximab did not improve 3 years RFS or OS. However, it was observed that RFS was longer in wild type group than in mutant group in both conventional and investigational (cetuximab) arms; conventional: $75.8 \%$ vs. $67.2 \%, \mathrm{P}=0.04$ and investigational: $72.3 \%$ vs. $64.2 \%$, $\mathrm{P}=0.004$ [25]. Limitations of our study in this context are: (I) small number of patients eligible for RFS analysis $(n=29)$ (II) there is mix of patients with stage II and III disease (III) some patients did while others did not receive adjuvant treatment (IV) relatively short duration of follow up (median 17 months). Difference in clinical outcome according to Kras status in patients with metastatic disease is also somewhat controversial. In one study comparing cetuximab with BSC, there was no difference in PFS and OS between mutant and wild type groups in the conventional arm. However, PFS and OS were shorter for the mutant type group in the investigational arm [10]. Similar trends were reported in 2 other studies comparing conventional chemotherapy with and without cetuximab [11, 12]. However, in another study comparing conventional chemotherapy with and without cetuximab, PFS and OS were shorter for mutant group than for wild type group in both treatment arms [13]. We will not be able to assess difference in outcome of patients with metastatic disease in our study due to small number and the fact that they used different treatment strategies.

In relatively small series from Asia including patients with all stages of disease (I-IV), the finding of the Chinese study concurs with our results showing no significant difference in OS between mutant and wild type groups [18]. On the other hand the Saudi study reports shorter OS in mutant group compared to wild type group [15].

\section{CONCLUSION}

This relatively small retrospective series shows that rate of K-ras mutation in Saudi patients with CRC is $32 \%$ which is lower than reported in western Caucasian population but close to rates reported in neighboring Asian and Middle Eastern population. Mutations are less frequent in females. Rates of codon 12 and 13 mutations are similar to those in other parts of the world. In these patients, K-ras mutation status did not significantly affect clinical outcome. More studies with larger cohorts are needed to establish ethnic and geographic variations in K-ras mutation from patients with CRC.

\section{TRIAL REGISTRATION}

This is not a clinical trial. This is a retrospective clinical study. Approval of institutional review board (ethics committee) has been obtained prior to conducting this study.

\section{ACKNOWLEDGEMENT}

None declared.

\section{CONFLICT OF INTEREST}

None declared.

\section{REFERENCES}

[1] Parkin DM, Bray F, Ferlay J, et al. Global cancer statistics, 2002. CA Cancer J Clin 2005; 55: 74-108.

[2] Kopetz S, Chang GJ, Overman MJ, et al. Improved survival in metastatic colorectal cancer is associated with adoption of hepatic resection and improved chemotherapy. J Clin Oncol 2009; 27: 3677-83.

[3] Chau I, Cunningham D. Treatment in advanced colorectal cancer: what, when and how? Br J Cancer 2009; 100: 1704-19.

[4] Porebska I, Harlozinska A, Bojarowski T. Expression of the tyrosine kinase activity growth factor receptors (EGFR, ERB B2, ERB B3) in colorectal adenocarcinomas and adenomas. Tumour Biol 2000; 21: 105-15.

[5] Salomon DS, Brandt R, Ciardiello F, et al. Epidermal growth factor-related peptides and their receptors in human malignancies. Crit Rev Oncol Hematol 1995; 19: 183-232.

[6] Cunningham D, Humblet Y, Siena S, et al. Cetuximab monotherapy and cetuximab plus irinotecan in irinotecan-refractory metastatic colorectal cancer. N Engl J Med 2004; 351: 337-45.

[7] Van Cutsem E, Kohne $\mathrm{CH}$, Hitre E, et al. Cetuximab and chemotherapy as initial treatment for metastatic colorectal cancer. N Engl J Med 2009; 360: 1408-17.

[8] Vogelstein B, Fearon ER, Hamilton SR, et al. Genetic alterations during colorectal-tumor development. N Engl J Med 1988; 319: 525-32.

[9] Ibrahim EM, Zekri JM, Bin Sadiq BM. Cetuximab-based therapy for metastatic colorectal cancer: a meta-analysis of the effect of $\mathrm{K}$ ras mutations. Int J Colorectal Dis 2010; 25(6): 713-21.

[10] Karapetis CS, Khambata-Ford S, Jonker DJ, et al. K-ras mutations and benefit from cetuximab in advanced colorectal cancer. N Engl J Med 2008; 359(17): 1757-65.

[11] Bokemeyer C, Bondarenko I, Hartmann JT, et al. Efficacy according to biomarker status of cetuximab plus FOLFOX-4 as first-line treatment for metastatic colorectal cancer: the OPUS study. Ann Oncol 2011; 22(7): 1535-46.

[12] Tol J, Koopman M, Cats A, et al. Chemotherapy, bevacizumab, and cetuximab in metastatic colorectal cancer. N Engl J Med 2009; 360(6): 563-72.

[13] Van Cutsem E, Köhne CH, Láng I, et al. Cetuximab plus irinotecan, fluorouracil, and leucovorin as first-line treatment for metastatic colorectal cancer: updated analysis of overall survival according to tumor KRAS and BRAF mutation status. J Clin Oncol 2011; 29(15): 2011-9.

[14] Vaughn CP, Zobell SD, Furtado LV, et al. Frequency of KRAS, BRAF, and NRAS mutations in colorectal cancer. Genes Chromosomes Cancer 2011; 50(5): 307-12.

[15] Abubaker J, Bavi P, Al-Haqawi W, et al. Prognostic significance of alterations in KRAS isoforms KRAS-4A/4B and KRAS mutations in colorectal carcinoma. J Pathol 2009; 219(4): 435-45.

[16] Sameer AS, Chowdhri NA, Abdullah S, et al. Mutation pattern of $\mathrm{K}$-ras gene in colorectal cancer patients of Kashmir: a report. Indian J Cancer 2009; 46(3): 219-25.

[17] Soliman AS, Bondy ML, El-Badawy SA, et al. Contrasting molecular pathology of colorectal carcinoma in Egyptian and Western patients. Br J Cancer 2001; 85(7): 1037-46.

[18] Shen H, Yuan Y, Hu HG, et al. Clinical significance of K-ras and BRAF mutations in Chinese colorectal cancer patients. World J Gastroenterol 2011; 17(6): 809-16.

[19] Segal G, Liebermann N, Klang S, et al. Identification of K-RAS mutations in colorectal cancer patients in Israel. Harefuah 2011; 150(5): 447-50.

[20] Doi T, Tahara M, Yoshino T, et al. Tumor KRAS status predicts responsiveness to panitumumab in Japanese patients with metastatic colorectal cancer. Jpn J Clin Oncol 2011; 41(2): 210-6. 
[21] Zalis MG, Vieira FM, Zalcberg-Renault I, et al. KRAS mutation profile in colorectal cancer patients in Brazil: A cohort of 989 individuals. J Clin Oncol 2009; 27 (Suppl): el5017.

[22] Zlatian O, Jalba CS, Ioana M, et al. Evaluation of activating mutations in codons 12 and 13 from exon 2 of KRAS oncogene in patients with colorectal adenocarcinoma. Ann Rom Soc Cell Biol 2010; 15(2): 174181.

[23] Zlobec I, Bihl MP, Schwarb H, et al. Clinicopathological and protein characterization of BRAF- and K-RAS-mutated colorectal cancer and implications for prognosis. Int $\mathrm{J}$ Cancer 2010; 15; 127(2): 367-80
[24] Ogino S, Meyerhardt JA, Irahara N, et al. Cancer and Leukemia Group B; North Central Cancer Treatment Group; Canadian Cancer Society Research Institute; Southwest Oncology Group. KRAS mutation in stage III colon cancer and clinical outcome following intergroup trial CALGB 89803. Clin Cancer Res 2009; 15(23): 73229.

[25] Goldberg RM, Sargent DJ, Thibodeau SN, et al. Adjuvant mFOLFOX6 plus or minus cetuximab (Cmab) in patients (pts) with KRAS mutant $(\mathrm{m})$ resected stage III colon cancer $(\mathrm{CC})$ : NCCTG Intergroup Phase III Trial N0147. J Clin Oncol 2010; 28:15.

(C) Zekri et al.; Licensee Bentham Open.

This is an open access article licensed under the terms of the Creative Commons Attribution Non-Commercial License (http://creativecommons.org/licenses/ by-nc/3.0/) which permits unrestricted, non-commercial use, distribution and reproduction in any medium, provided the work is properly cited. 